Revue d'histoire de l'Amérique française

RAS REVUE D.HISTOIRE DE L'AMÉRIQUE FRANÇAISE

\title{
Inventaire des documents concernant l'histoire du Canada conservés aux Archives de Chicago (suite)
}

\section{Honorius Provost}

Volume 4, numéro 3, décembre 1950

URI : https://id.erudit.org/iderudit/801663ar

DOI : https://doi.org/10.7202/801663ar

Aller au sommaire du numéro

Éditeur(s)

Institut d'histoire de l'Amérique française

ISSN

0035-2357 (imprimé)

1492-1383 (numérique)

Découvrir la revue

Citer ce document

Provost, H. (1950). Inventaire des documents concernant l'histoire du Canada conservés aux Archives de Chicago (suite). Revue d'histoire de l'Amérique française, 4(3), 453-458. https://doi.org/10.7202/801663ar d'utilisation que vous pouvez consulter en ligne. 


\title{
BIBLIOGRAPHIE
}

\section{INVENTAIRE DES DOCUMENTS CONCERNANT L'HISTOIRE DU CANADA CONSERVÉS AUX ARCHIVES DE CHICAGO}

\author{
(suite)* \\ $-\mathrm{II}-$
}

\section{SOCIÉTÉ HISTORIQUE DE CHICAGO}

Adresse: The Chicago Historical Society, Lincoln Park, Chicago, $\mathrm{Il}$.

1625, avril 28. Reçu signé à Paris par de Lauzon.

1626, avril 22. Acceptation d'un contrat, signé "Anthoynette de Pons", marquise de Guercheville.

1644, juin 12. Requête au Gouverneur par Guillaume Couillard pour une assemblée de parents des mineurs de feu Guillaume Hébert et de Hélène Desportes, remariée à Noël Morin, à Québec (x).

1644, juin 12. Autorisation accordé à la pétition de G. Couillard, sign $6 \Theta$ à Québec par Montmagny. Procès-verbal de l'assemblée de parents (x).

1644, août 16. Concession par Montmagny, pour la Cie de la N. France, à Guillaume Isabelle, aux Trois-Rivières.

1649, mars 29. Approbation de la concession ci-dessus par la Cie de la N.France. Parchemin signé à Paris par Lamy.

1650 , juin 9. Permission touchant une certaine propriété des Jésuites, signée aux Trois-Rivières par le P. Jérôme Lalemant.

1653, jan. 9. Fragment d'une pétition au gouverneur signé par les habitants des Trois-Rivières $(x)$.

1653, oct. 19. Remise de droits seigneuriaux par le P. Léonard Gareau. 1653, nov. 20. Concession de terrain par Jean de Lauzon.

1656, juin 20. Concession de terrain près du Fort de Québec, par Jean de Lauzon.

1656, juil. 4. Concession de terrain près des Trois-Rivières par le P. Jacques de La Place.

* Voir Revue d'Histoire de l'A mérique française, (sept. 1950): 294-302.

(x) Les documents ainsi marqués ont été reproduit plus ou moins fidèlement dans le Rapport des Archives Canadiennes (1905), v. 1, au début. 
1657, sept... Fragment d'une pétition signée par les habitants de VilleMarie (Montréal) (x).

1659, mai 19. Médard Chouart; quittance en faveur de Christophe Crevier, Sieur de la Meslé; présent, François Maistrə.

1660, mai 24. Contrat de mariage de Pierre Bissonnet, menuisier, et Mathurine Desbordes, à Ville-Marie. Minute du not. Basset. Signatures de Maisonneuve, D'Ailleboust, Coulonge, LeGardeur, etc.

1661, juin 8. Procuration à Pierre Boucher pour régler une dispute, signée par Claude Allouez, S.J. aux Trois-Rivières (x).

1661, déc. 7. Concession par les Jésuites à Médard Chouart, au Capde la Madeleine; signéө "Médard Chouart"; minute de notaire (Ameau ?) des Trois-Rivières. Au verso, fragment d'un contrat de mariage, du 19 nov. 1661 (document très effacé, qui devra être photographié avec soin).

1661, ... Fragment de document portant les signatures du R.P. Claude Allouez, de Jean Guyon, Nicolas Gaillon, Jean Cusson et Moral de StQuentin.

1662, mai 15. Reconnaissance d'un prêt d'argent fait par le P. Claude Allouez, Trois-Rivières, à Médard Chouart, par sa femme (x).

1663, mars 28. Concession par le P. Claude Allouez à Jérôme Langlois, d'un emplacement “aux Trois-Rivières dans l'enclos du bourg".

1663, avril 20. Donation par la veuve de François Fouquet à l'Hôpital de Québec de la huitième partie de la seigneurie de Beaupré et de l'Ile d'Orléans; minute du not. Vallon(?), à Paris; signature du Père Paul Raguoneau, pour l'Hôtel-Dieu.

1663, ... Ordre judiciaire de procéder à la vente de certains effets; signe par LeBer, Lemoyne, etc.

1665, avril 20. Contrat entre Jacques Gouillet et Benjamin Cazeau. 1665, juin 13. Engaigement, par procuration de Jean Gladu, en fatveur de Louis Beaudry; signe de François Bibeau et Nicolas Dupuy.

1665(?), août 4. Contrat signé pair Maisonneuve, la vve d'Ailleboust, Ch. de Musseaux, etc.

1665, nov. 10. Fragment apparemment d'une quittance, signê de Simon Lemoyne, S.J., Moral de St-Quentin, Pierre Lefebvre, etc.

1666, avril 20. Acte de société entre Adrien Jolliet, Denys Guyon et d'autres (x).

1667, aout 12. Acte de societé entre Jean Desroches et Isaac Nafrechon, d'une part, et Toussaint Beaudry et Nicolas Perrot, d'autre part.

1667, oct 11. Reçu en faveur de Laurent Lefebvre, signé par le P. Chàrles Albanel.

1668, mars 9. Concession sous seing privé à René Cuillerier, à la Côte St-Sulpice, signee par R. Cavelier de la Salle.

1668, mai 12. Fragment d'un arbitrage par de Varennes, Jacques LeMoyne et Michel Messier: un terrain sur le fleuve, près la rivière NotreDame, vis-à-vis les îles Bourgaud. Signé Courcelle et d'Ailleboust. 
1668, juin 22. Accord entre Barbe de Boulogne, veuve de Louis D'Ailleboust, et son neveu Charles D'Ailleboust, sieur de Musseaux, concernant les biens de la succession $(x)$.

1668, juin 27. Mémoire de Jean Talon touchant la requête du Sr de Musseaux pour concession de l'Ile Bourdon et deux autres îles (x).

1669, janv. 18. Engagement signé par Jacques Maugras, Nicolas le

Bossu et Martin Carpentier, pour un voyage de traite.

1670, avril 6. Ordre à Laurent Lefebvre de payer à Lavigne, par le R.P. J. Richard, S.J.

1670, avril 26. A Paris, obligation du marquis de Tracy et de son épouse à Henri de la Fresnaie, leur écuyer (x).

1671, déc. 26. Reçu de Rasnuyer en faveur de Marin Hurtubise. lus (x).

1672, juin 18. Concession à Jean Boutin dit Léveillé, par M. de Quey-

1672, sept. 14. Ordonnance signée par Jean Talon à toutes personnes de lui déclarer leurs créances avant son départ (x.) LaRue.

1672, oct. 5. Certificat de publication de l'ordonnance du 14 sept. signé

1672 (circa). Concession de terrain sur le fleuve au Sr Lemoyne; signê Courcelles, contresignée P. D’Ailleboust.

1673, avril 25. Ratification d'une concession à M. D'Ailleboust, faite sur billet par le P. LeMercier; signée Claude Dablon (x).

1674, avril 25. Concession signée Frontenac au Sr de St-Ours, des îles devant sa seigneurie; contresignée Lechasseur.

1674, juin 3. R.P. Louis Nicolas, S.J.; concession à André Dubois. 1674, juil. 7. Poursuite de Mathurin Beausoleil, époux de la veuve Adrien Jolliet, contre Louis Jolliet. Ordre à ce dernier de produire une copie du mémoire "pour remplacer celui qu'il dit avoir perdu dans son dernier naufrage". Jugement dans cette cause rendu par Louis Rouer de Villeray, Thierry de l'Estre et LeVallon; volumineux dossier.

1675, mai 2. Quittance sur parchemin pour 6,000 livres, signée à Paris par le Comte d'Estrées, vice-roi de N. France.

1675, mai 13. Lettres de noblesse pour Robert Cavelier de La Salle et certificat de leur enregistrement (x).

1675, sept. 23. Concession par le Roi à R. Cavelier de La Salle du Fort Frontenac et des îles et terres environnantes. Certificat d'enregistrement à Québec, signé Peuvret.

1676, juillet. Lettre du chirurgien Jean Martinelle dit Fontblane à l'intendant Duchesneau, concernant la succession du chirurgien Etienne Bouchard.

1676, ... Procès-verbal d'un procès pour assaut, devant Rageot, où paraissent comme témoins Louis Jolliet, la vve Bissot et autres.

1678, ... Ratification d'accord signée Pierre LeMoyne d'Tberville.

1679, juin 2. Concession de terrain à la Prairie de la Madeleine à Jacques Perras, par le Père Jacques Fremin, S.J. 
1680, fêv. 19. Contrat de mariage entre Jean Marsollet et Marguerite Couture, fille de Guillaume Couture; signatures de Frontenac et Duchesneaux (x).

1680, avril 14. Vente par les hér. Bissot à Volant de St-Claude d'une propriété à la Basse-Ville de Québec. Signature de Louis Jolliet.

1681, mars. Autorization par Duluth au Sr Hébert, concernant la traite à Michilimakinac.

1682, mars 24. Reçu signé par Abraham Brouat.

1682, mai 20. Marché pour une expédition au pays des Outaouais, entre Hazeur, de Monseignac, Zacharie Jolliet, Lambert Leduc; Boucher, notaire à Montréal.

1682, août 12. Paiement fait au Sault Ste-Marie par M. de Bérancourt en faveur de MM. d'Argenteuil et Coulonge (x).

1682, sept. 12. Marché à Montréal pour un vuyage à Michilimakinac, entre M. LeSueur et autres.

1682, déc. 7. Concession sous seing privé par "De La Salle” à Chs Dizé, d'un terrain au Fort St-Louis, Mississipi.

1683, avril 26. Contrat de concession par Robert Cavelier de La Salle

à Jacques Bourdon (x).

1683, mai 8. Ordre d'appeler des témoins devant Migeon, à Montréal; signé de LaBarre.

1683, mai 8. Requête au Gouverneur par Louis Juillet, de Montréal pour remboursement par le Sr de LaValtrie. Affaire soumise à l'Intendant; signé de La Barre.

1683, sept. 1. Lettre autographe de R. Cavelier de La Salle, datée de Chicago, aux occupants du Fort St-Louis de Illinois (x).

1683, déc. 1. Marché pour construction de maison par Claude Baillif à Louis Jolliet, à Québec; entièrement de la main de ce dernier. Déposé dans le greffe de Rageot, le 4 sept. 1684 (une copie en photostat se trouve aux archives judiciaires de Québec).

1684, fêv. 21. Lettre signée à Québec, par "De Comportê", concernant un terrain qu'il a fait vendre à l'enchère.

1684, oct. 3. Marché de René Allary, charpentier, avec François Couillard de la Fontaine, pour une maison à la haute-ville de Québec (photostat de l'original, qui se trouve au musée McCord, à Montréal).

1684, oct. 15. Engagement devant un notaire de Montréal de J.Bte Nolan et Maurice Blondeau au Sieur de Tonty, qui signe (x).

1684, oct. 15. Engagement semblable, même endroit, signê Tonty (x).

1684, oct. 21. Requête en restitution de propriété adressée au Sr Gervaise, par Marguerite Nicollet et Louise Bissot. Requête accordée; signée Jehan Gervaise (x).

1685, mars. Contrat de traite entre Claude Tessier et Claude Grizonneau. taine.

1685, juil. 16. Jean Roy, Michillimakinac, à Vincent Dugas dit Lafon- 
1685, sept. Engagement de Paul Bouchard, Montreal, au Sr François Rolland pour un voyage aux Illinois.

1685, ... Concession de terrain à Jacques Cardinal, signée Tonty (x).

1686, août 18. Obligation de Lépine à Jacques Bourdon, notaire Turpin(?), Montréal.

1686, août 19. Mémoire des marchandises livrées à Pierre Morin par le Sr. Millot, pour une expédition aux Illinois.

1686, mai 19. Reçu signé par le R.P. Thierry Beschefer, S.J. au Cap de la Madeleine.

1686, nov. 1. Lettre au Sr Duguay de Boisbriant, par Lafleur, auparavant sergent sous ses ordres au Fort Frontenac.

1686, nov. 2. Lettre datée de Lachine, à Delle Madeleine de Roybon d'Allonne, au Fort Frontenac, donnant des nouvelles de LaSalle, ecrite par Joseph Michaud (x).

1687, juin 7. Vente par Duguay de Boisbriant, au nom de Lafleur, à Nicolas Chapput, d'une ferme dans la seigneurie de l'Ile Ste-Thérèse.

1687, août 26. Concession par M. de St-Ours aux habitants de Chambly, d'un terrain en commun; signée à Ville-Marie par Denonville et Champigny. 1688, juillet. Marché pour la traite avec les sauvages, entre Jean Lepellé, Jacques Cardinal et autres, à Montréal.

1688, août 3. Facture de marchandises de traite livrées à François Côté dit Lépinard, Joseph Pelletier et Et. Michel.

1688 , oct. 16. Déclaration signée à Boucherville par Pierre Boucher et son épouse, exonérant le Sr LeVerrier de tout blâme pour la mort accidentelle de lour fils (x).

1689, janv. 20. Reconnaissance de compte par René Cuillerier.

1689, juin 1. Lettre de l'abbé Ls Tronson à M. de Blainville, capitaine à Montréal (x).

1689, juin 5. Certificat d'aptitude à M. de la Durantaye.

1689, juil. 11. Fragment de lettre signée par Bochard de Champigny, à Villo-Marie.

1689, oct. 15. Certificat de mérite par le Marquis de Denonville, à Villo-Marie, en faveur du Sr de Grandpré, enseigne dans la compagnie de M. de Lorimier.

1689, nov. 5. Reçu par Bertrand Arnaud en faveur de Jean Mongeon.

1690, fév. 2. Lettre de René Cuillerier, du Fort Cuillerier, à Bourgine Peuvret, procureur fiscal à Montréal, au sujet de Louis Fortier.

1690, mai 15. Déposition de Frs. de Boisrondet sur ce qui est survenu durant un voyage; compagnon de La Salle et de Tonty.

1690, mai 15. Reçu en faveur de Nicolas Perrot, par Charles Amiot, autorisé à agir pour Catignon, Charles Patu et les fermiers de Michillimakinac.

1691, avril. Reconnaissance de dette par Alexandre Turpin, fils, pour vente de fourrures apportées de son voyage aux Outaouais, avec Nicolas Perrot. 
1691, juil. 20. Marché de traite, à Michillimakinac, entre Pierre Chesne, dit St-Onge, et Pierre Chartier.

1691, juil. 23. Congé de traite aux Outaouais pour le Sr de la Durantaye, par Frontenac (x).

1691, juil. 26. Congè à Macon pour Michillimakinac, par Frontenac (x). 1691, juillet. Billet promissoire de Jacques Bourdon, Sieur d'Autray, en faveur de Michel Morin dit Lafontaine.

1691, août 1. Reçu de Louis Duquet en faveur de Nicolas Perrot; témoins: Ls Cavelier et Nicolas Laurent, à Michillimakinac.

1691, août 27. Marché de traite entre le Sr de la Durantaye et Jean Fafard dit Macon, à Michillimakinac (x).

1692, mars 17. Obligation de Arnaut de Godefroy à Delle de Lamarque; signature de Hertel.

1692, août 15. Lettre autographe de Dominique de LaMothe.

1693, avril 19. Vente d'une terre en Louisiane, par François de La Forest à Michel Alan, pour être payée à Chicago.

1693, mai 3. Difficultés à propos d'un bail; J.Bte Migeon de Bransat, juge à Montréal.

1693, sept. 11. Contrat à Ville-Marie entre le Sr de Courtemanche et Médard Mézéré. Salle). 1693, ... Accord signé par Moìse Hilaret (chef des mutins contre La 1694, ... Rapport de l'expédition à Michillimakinac.

1695, juil. 2. Marché de traite, à Michillimakinac, entre Macon et Jean Beauvais, au service de M. de La Mothe, d'une part, et les Srs Moreau et Lépine; signé par Jean Roy et Jean Fafart.

1695, août 1. Accord au sujet de la traite à Michillimakinac par Nicolas Laurent dit Lachapelle.

1695, août 8. Accord au sujet de la traite entre François de BoisGuillot et Pierre Lesueur.

1695, nov. 8. Contrat de société entre Louis Jolliet et Claire Bissot, son épouse, avec Charles et François Bissot, pour usage des Iles de la côte de Mingan. Signature de Jolliet, etc. (Les Archives de la Province ont aussi cette pièce).

$1696, . .$. Lettre du Baron de Longueil au sujet d'un nommé St-Martin. 1697, fév. 17. Procuration par Nicolas Perrot à ses trois fils (x).

1697, juil. 21. Billet promissoire en faveur de Ls de La Salle par de la Forest.

1697, sept. 6. Engagement de Pierre Lepellé par Jean Bourdon pour un voyage aux Outaouais.

1697, ... Déclaration en faveur d'un déserteur, par l'abbé de La Salle. 1698, juil. 23. Obligation devant notaire de Joseph Charbonneau, Montréal, à Pierre You.

1698, sept. 23. Congé de traite au Sr de la Forest et à Tonty, signé Frontenac et Champigny (x).

(à suivre)

Honorius Provost, ptre 